\title{
The association between hyperandrogenemia and the metabolic syndrome in morbidly obese women
}

\author{
T.G. Valderhaug ${ }^{1,2,3^{*}}$, J.K. Hertel ${ }^{1}$, N. Nordstrand ${ }^{1}$, P.O. Dale ${ }^{4}$, D. Hofs $\varnothing^{1}$ and J. Hjelmesæth ${ }^{1,5}$
}

\begin{abstract}
Background: Female abdominal obesity is associated with hyperandrogenemia (HA), but few studies have addressed the possible association between HA and metabolic syndrome (MetS) among obese women. Some studies indicate that insulin resistance may cause HA through different mechanisms. On the other hand, a bidirectional relationship between HA and insulin resistance has been suggested. Thus, we aimed to investigate if morbidly obese women with HA had higher odds of MetS and its components than those without HA (controls), independent of polycystic ovarian syndrome (PCOS) status.
\end{abstract}

Methods: This cross-sectional study comprised 1900 consecutive treatment seeking morbidly obese women $<50$ years. Free testosterone index $(\mathrm{FTI})>0.6$ defined HA. Women with previously diagnosed PCOS and those with oligo- / anovulation combined with clinical or biochemical hyperandrogenism were defined as having PCOS. Multiadjusted associations between HA and MetS were assessed by logistic regression analysis.

Results: Out of 1900 morbidly obese women, 1089 (57 \%), 846 (45 \%) and 312 (16\%) had MetS, HA and PCOS, respectively. Compared with controls (without HA), women with HA were younger (34 [1] years vs. 39 [2], $p<0.001$ ) had a higher prevalence of MetS ( $62 \%$ vs. $53 \%, p<0.001$ ), type 2 diabetes (18\% vs. $15 \%, p=0.045$ ), low HDL-cholesterol ( $65 \%$ vs. $48 \%, p<0.001$ ) and hypertriglyceridemia ( $48 \%$ vs. $41 \%, p=0.004$ ), but a lower prevalence of raised blood pressure (53 \% vs. $59 \%, p=0.014)$. Multivariable analyses showed that HA was associated with increased odds of MetS (OR 1.61 [95 \% Cl 1.27, 2.02]), dysglycemia (1.65 [1.28, 2.11]), low HDL-cholesterol (1.58 [1.27, 1.97]), and hypertriglyceridemia $(1.43[1.15,1.79])$. After stratification for the presence of PCOS, the results remained largely unchanged in women without PCOS; MetS $(1.52[1.18,1.96)$, dysglycemia $(1.71[1.30,2.25])$, low HDL-cholesterol (1.55 [1.22, 1.98]) and hypertriglyceridemia (1.36 [1.06, 1.74]).

Conclusion: Morbidly obese women with HA had an approximately 1.5-fold increased odds of having MetS even in the absence of PCOS. Randomized controlled clinical trials, including therapeutic strategies to lower free testosterone levels, are however necessary to explore any cause-and-effect relationship.

Keywords: Morbid obesity, Insulin resistance, Hyperandrogenemia, Metabolic syndrome

\footnotetext{
* Correspondence: t.g.valderhaug@medisin.uio.no

'Morbid Obesity Centre, Vestfold Hospital Trust, Tønsberg, Norway

${ }^{2}$ Division of Medicine, Department of Endocrinology, Akershus University

Hospital HF, Sykehusveien 25, 1478 Nordbyhagen, Norway

Full list of author information is available at the end of the article
} 


\section{Background}

The polycystic ovarian syndrome (PCOS) is the most common female endocrinopathy, affecting $8-12 \%$ of women of reproductive age [1], and 10-35\% of obese women [2-4]. PCOS is associated with infertility, hyperandrogenemia, impaired glucose tolerance and type 2 diabetes [5]. A combination of increased levels of androgens and insulin is believed to contribute to the pathophysiology of PCOS [5]. Hyperandrogenemia (HA) comprises the biochemical hallmark of PCOS with elevated free testosterone levels accounting for the majority of the abnormal laboratory findings in women with oligomenorrhea [6].

Excess body weight is associated with HA [7]. Furthermore, the hyperinsulinemia in obese women may directly increase free testosterone levels by lowering the sex hormone binding globulin synthesis in the liver [8]. On the other hand, rodent models have shown that HA promotes insulin resistance, reduces energy expenditure and, accordingly, increases the risk of abdominal obesity and metabolic risk factors $[9,10]$. In a multiethnic sample of more than 2500 U.S. women between 42 and 52 years of age, oligomenorrhea was associated with the metabolic syndrome (MetS) only when coincident with HA [11]. Conversely, women with HA had a significantly increased risk of the MetS independent of the menstrual frequency status [11].

The ovaries' response to luteinizing hormone $(\mathrm{LH})$ is the main source of increased androgens in PCOS [12]. Also, the elevated response of adrenal steroids in women with PCOS sustained only with adrenal stimulation of $\mathrm{ACTH}$, suggesting a secondary effect of increased adrenal androgen production rather than adrenal congenital enzyme deficit [13].

It is not clear how HA may affect cardiovascular disease. Abdominal obese women with PCOS are considered at high risk of cardiovascular disease, and a positive association between coronary artery disease and clinical hyperandrogenism (hirsutism and acne) has been reported [14]. On the other hand, a population based study showed that premenopausal overweight women with PCOS did not have a higher risk of coronary artery disease than those without PCOS [15]. Nevertheless, women with PCOS and HA have a higher prevalence of obesity and adverse metabolic abnormalities compared to those without HA [16]. To the best of our knowledge, no previous study has assessed the impact of HA on the MetS independent of the presence or absence of PCOS among obese women.

The primary aim of this study was to examine whether treatment seeking premenopausal morbidly obese women with HA had higher odds for MetS and its components (low HDL-cholesterol, hypertriglyceridemia, raised blood pressure, and dysglycemia) than women without HA, independent of PCOS status.

\section{Methods}

\section{Design and study population}

A total of 2681 consecutive treatment seeking morbidly obese women attending the Morbid Obesity Centre at Vestfold Hospital Trust, Norway, during the period from November $28^{\text {th }} 2005$ until July $28^{\text {th }} 2014$ were assessed for eligibility. To avoid biological bias of including both pre- and postmenopausal women, we excluded 743 women $\geq 50$ years [17-19]. Data on HA were missing for 38 women (PCOS absent $\mathrm{n}=34$ and PCOS present $\mathrm{n}=4$ ), leaving 1900 morbidly obese women of reproductive age to be included in this cross-sectional analysis. The study was approved by the Regional Committee for Medical and Health Research Ethics (S-05175). The participants were included after providing written informed consent, and the study was performed in accordance with the Declaration of Helsinki [20].

\section{Definitions}

We defined MetS according to the joint interim statement of the International Diabetes Federation Task Force on Epidemiology and Prevention; National Heart, Lung, and Blood Institute; American Heart Association; World Heart Federation; International Atherosclerosis Society; and International Association for the Study of Obesity (2009); if WC $\geq 80 \mathrm{~cm}$ combined with a minimum of two out of four criteria present: 1) low HDLcholesterol; HDL-cholesterol $<1.3 \mathrm{mmol} / \mathrm{L}, 2)$ hypertriglyceridemia; triglycerides $\geq 1.7 \mathrm{mmol} / \mathrm{L}, 3$ ) raised blood pressure; systolic blood pressure $\geq 130 \mathrm{mmHg}$ or diastolic blood pressure $\geq 85 \mathrm{mmHg}$, or use of blood pressure lowering medication, and 4) dysglycemia; fasting serum glucose $\geq 5.6 \mathrm{mmol} / \mathrm{L}$ or diabetes mellitus [21]. Type 2 diabetes was diagnosed in patients who had a prior history of type 2 diabetes or a fasting plasma glucose $\geq 7.0 \mathrm{mmol} / \mathrm{L}$ or an HbA1c $\geq 6.5 \%$. The Homeostasis Model Assessment Insulin Resistance (HOMA IR) was calculated as ([fasting serum glucose (mmol/l) * fasting serum insulin (pmol/l)]/135) [22]. We calculated the free testosterone index (FTI) using the formula: $\mathrm{FTI}=100 \mathrm{x}$ serum testosterone $(\mathrm{nmol} / \mathrm{L}) /$ sex hormone binding globulin (SHBG, nmol/L). Free testosterone was calculated from the formula described by Vermeulen et al. [23]. According to the Androgen Excess \& PCOS Society consensus statement on PCOS diagnosis, HA is defined by increased levels of free testosterone [6, 24]. Accordingly, women with an FTI above the upper normal range (FTI $>0.6$ ) were defined as having HA [25]. Women with previously diagnosed PCOS and those with an FTI $>0.6$ or hirsutism combined with oligo- / anovulation were defined as having PCOS [16, 26]. 


\section{Data collection}

Patients had their weight and height measured wearing light clothing, without shoes, and BMI was subsequently calculated $\left(\mathrm{kg} / \mathrm{m}^{2}\right)$. We measured WC midway between the lowest rib margin and the iliac crest. Blood pressure was measured with an appropriate cuff after at least 5 minutes rest with the patient seated in an upright position. Three measurements were registered and the average of the second and the third measurement was used in the study. All anthropometric and blood pressure measurements were performed by trained study personnel.

\section{Laboratory analysis}

Blood samples were obtained by venipuncture after an overnight fast and collected in Vacutainers ${ }^{\circ}$ gel tubes. Serum was separated from cells within two hours.

Analyses of serum glucose, magnesium, uric acid, and blood lipids were performed using dry reagent slide technology on the Vitros 950 Analyzer until November 2006 and thereafter on the Vitros FS 5.1 (Ortho-Clinical Diagnostics, New York, USA). Intact PTH was measured using an electro-chemiluminescence immunoassay on the Elecsys 2010 (Roche Diagnostics $\mathrm{GmbH}$ ) or Architect i1000SR (Abbott Diagnostics). The results of the electro-chemiluminescence immunoassay were multiplied by a conversion factor of 1.34 [27]. Glycosylated hemoglobin was analyzed by high performance liquid chromatography (HPLC) using Tosoh HLC-723 G7 (Tosoh Corporation, Tokyo, Japan). All other analyses were performed on the day of blood sampling at the Department of Clinical Chemistry at Vestfold Hospital Trust. Sera for analysis of insulin, testosterone, SHBG and $25(\mathrm{OH})$ vitamin D (25-hydroxyvitamin $\mathrm{D}_{2}+25$-hydroxyvitamin $\mathrm{D}_{3}$ ) were stored at $-20{ }^{\circ} \mathrm{C}$ and analyzed within one week of blood sampling at the Hormone Laboratory, Oslo University Hospital, Aker. Insulin and $25(\mathrm{OH})$ vitamin D were analyzed in serum by radioimmunoassay (Linco Research Inc, St Charles, MO, and DiaSorin, Stillwater, $\mathrm{MN})$. The interassay coefficients of variation (CV) for insulin and $25(\mathrm{OH})$ vitamin D were $8 \%$ and $14 \%$, respectively. Testosterone was measured in serum by a competitive radioimmunoassay (RIA) technique using ${ }^{125} \mathrm{I}$ testosterone (Orion Diagnostica, Espoo, Finland) with a coefficient of variance $(\mathrm{CV})$ at the lower normal reference interval (mean $2.2 \mathrm{nmol} / \mathrm{L}$ ) of $11 \%$. Samples with a testosterone concentration level $\geq 4 \mathrm{nmol} / \mathrm{L}$ were checked for interference after ether extraction. SHBG in serum was measured by immunoluminometric assay (ILMA) (Immunlite 2000 or 2500, Siemens, NY, US, Healthcare Diagnostics) with a $\mathrm{CV}$ of $6 \%$.

\section{Statistical analysis}

Data are presented as mean (SD) or proportions. Continuous and categorical variables were compared using independent samples t-test and $\chi^{2}$ test or Fisher's exact test as appropriate. Correlations were calculated with Pearson's correlations coefficient (r) for normally distributed variables and Spearman's Rho ( $\rho)$ for non-normally distributed variables. Demographic, anthropometric and metabolic variables were analyzed including the whole study population and after stratification for presence or absence of PCOS.

The MetS and its components were modeled as dependent variables and HA as the primary explanatory variable. The association between $\mathrm{HA}$ and MetS was adjusted for significant confounders identified using a backward logistic regression approach. Variables with p-values below 0.10 were included in the final model (Wald test). Consequently, coronary artery disease (yes/no), prednisolone usage (yes/no), chronic obstructive pulmonary disease (yes/no), joint pain (yes/no), use of estrogens or gestagen medication (yes/no), and thyroid stimulating hormone were removed from the regression model. The models were adjusted for the following covariates in the final multivariable analysis: age (years), family history of diabetes (yes/no), uric acid $(\mu \mathrm{mol} / \mathrm{L})$, anxiety or depression (yes/no), cholelithiasis (yes/no), history of smoking (current or former/never), physical activity ( $\geq 1$ hour vigorously physical activity/week), parathyroid hormone $(\mathrm{pmol} / \mathrm{L})$, and vitamin $\mathrm{D}-25-\mathrm{OH}$ (nmol/L). We decided not to adjust for WC and HOMA IR in order to avoid the possibility of overadjustment bias by variables known to be in the causal pathway between HA and the MetS [10, 28].

Univariate and multivariate logistic regression models were used to assess the associations between $\mathrm{HA}$ and MetS and its components (hypertriglyceridemia, low HDL-cholesterol, raised blood pressure, and dysglycemia) in all the 1900 included patients. In addition, the analyses were repeated in a subgroup of obese women without PCOS and another group with PCOS, respectively. Possible effect modifications by age and PCOS were investigated by including the products age" $\mathrm{HA}$, and $\mathrm{PCOS}^{*} \mathrm{HA}$ as interaction terms in the multivariable analyses with MetS as the dependent variable.

The goodness of fit was tested using the Hosmer and Lemeshow test. P-values below 0.05 were considered statistically significant. However, due to the considerable number of statistical tests performed, particular attention should be directed towards smaller P-values, i.e. those below 0.01. The analyses were implemented using IBM SPSS statistics 20. 


\section{Results}

A total of 1089 (57\%), 846 (45\%) and 312 (16\%) out of 1900 morbidly obese women under 50 years of age (mean age 37 [SD 8] years) had MetS, HA and PCOS, respectively. A total of 1445 women had either the metabolic syndrome (MetS), hyperandrogenemia (HA) or polycystic ovarian syndrome (PCOS), whereas 455 women had neither MetS, HA or PCOS. The overlap of women with MetS, HA and PCOS is presented in Fig. 1.

In the whole study population, women with HA were younger (mean [SD] age 34 [1] vs. 39 [2] years, $p<0.001$ ), heavier [body weight 125 [19] vs. 121 [18] $\mathrm{kg}, p<0.001$ ), had a higher WC (127 [13] vs. 123 [13] cm, $p<0.001)$, and were more insulin resistant than the women without HA (HOMA IR; 5.8 [5.5] vs. 4.5 [4.3], $p<0.001$ ). The HA group had a significantly higher prevalence of MetS (62\% vs. $53 \%, p<0.001)$, PCOS (27 \% vs. $8 \%, p<0.001)$ and were more likely to have low HDL-cholesterol (65 \% vs. $48 \%, p<0.001$ ), hypertriglyceridemia ( $48 \%$ vs. $41 \%, p=$ 0.004 ) and type 2 diabetes (18\% vs. $15 \%, p=0.045$ ). Women with HA had a lower prevalence of raised blood pressure (53\% vs. $59 \%, p=0.014$ ) and were less likely to use blood pressure lowering medication (18\% vs. $25 \%, p<0.001)$. A tendency towards a higher prevalence of dysglycemia was seen in women with HA compared to women without HA ( $32 \%$ vs. $28 \%, p=0.069$ ).

The demographic, anthropometric and metabolic differences between participants with and without HA did not change substantially after stratification according to presence or absence of PCOS (Table 1). The prevalence of MetS was higher in women with HA than in women without HA independent of the presence or absence of PCOS (PCOS absent: $60 \%$ vs. $53 \%, p=0.007$ and PCOS present: $68 \%$ vs. $55 \%, p=0.032$ ).

The proportion of women with HA decreased from $80 \%$ (<20 years) to $30 \%$ (40-50 years) with increasing age deciles (Fig. 2). By contrast, the percentages of women with MetS, dysglycemia, hypertriglyceridemia and raised blood pressure increased significantly with age (all $p<0.001$ ) (Fig. 2). The proportion of women with low HDL-cholesterol did, however, not change significantly with age $(p=0.145)$.

The FTI correlated weakly with HDL-cholesterol, systolic blood pressure and diastolic blood pressure $(r=$ $0.10, p<0.001 ; r=-0.05, p=0.040$; and $r=-0.06, p=$ $0.007)$ and HOMA IR $(\rho=0.22, p<0.001)$, but not with BMI, WC, triglycerides and fasting plasma glucose $(r=$ $0.04 ; r=0.03 ; r=0.03 ; r=-0.01$, all $p>0.111$ ). We did not find any significant interactions between $\mathrm{HA}$ and age $(p=0.834)$ or between HA and PCOS $(p=0.527)$.

In the univariate analysis, HA was associated with a 1.5 fold increased odds of MetS (OR 1.45 [95 \% CI 1.20, 1.74]). This association remained statistically significant after adjustments for possible confounders (1.61 [1.27, 2.02]) (Table 2 and Fig. 3A). Moreover, HA was associated with approximately 1.5 fold increased adjusted odds of dysglycemia $(1.65[1.28,2.11])$, low HDL-cholesterol $(1.58$ $[1.27,1.97])$, and hypertriglyceridemia $(1.43[1.15,1.79])$.

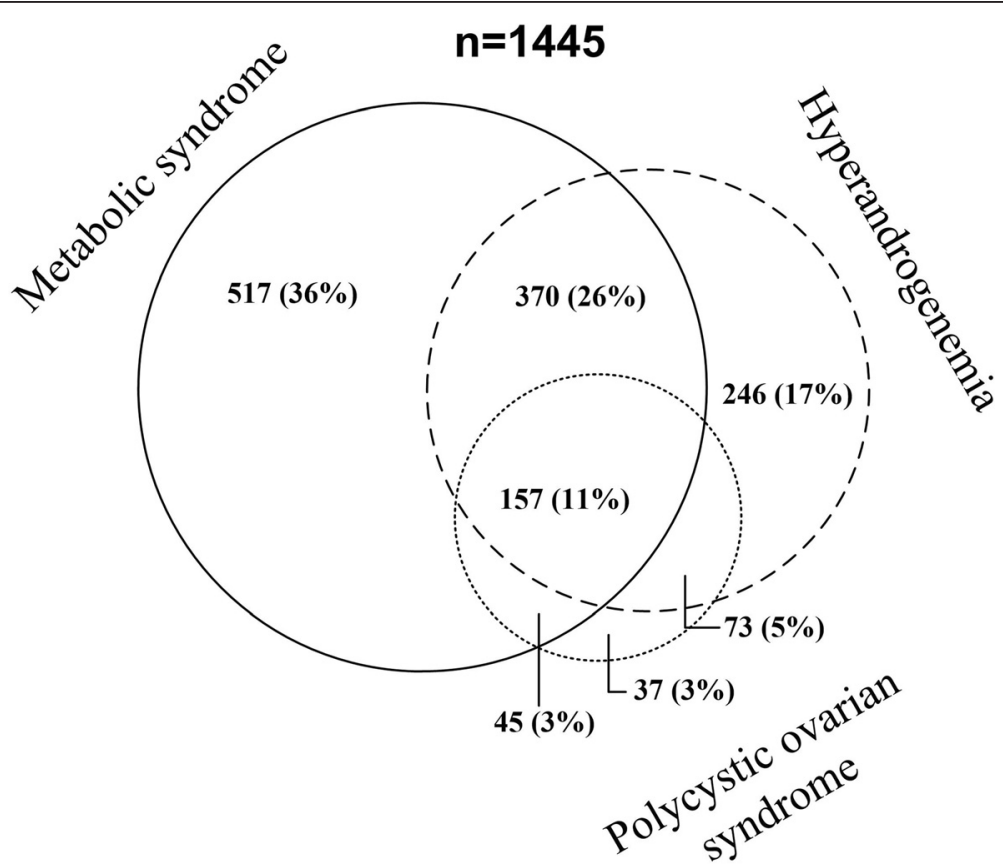

Fig. 1 The figure shows the overlap between the 1445 women characterized by their having the metabolic syndrome, polycystic ovarian syndrome and hyperandrogenemia (HA). Women without any of these conditions $(n=455)$ were not included in Fig. 1 
Table 1 Patient characteristic according to presence or absence of polycystic ovarian syndrome (PCOS) and hyperandrogenemia (HA)

\begin{tabular}{|c|c|c|c|c|c|c|}
\hline & \multicolumn{3}{|l|}{ PCOS absent } & \multicolumn{3}{|l|}{ PCOS present } \\
\hline & HA present & HA absent & $P$ value & HA present & HA absent & $P$ value \\
\hline $\mathrm{N}$ & 616 & 972 & - & 230 & 82 & - \\
\hline Age, yrs & $35(8)$ & $39(7)$ & $<0.001$ & $33(8)$ & $36(8)$ & 0.001 \\
\hline $\mathrm{BMI}, \mathrm{kg} / \mathrm{m}^{2}$ & $44.4(5.8)$ & $43.2(5.9)$ & $<0.001$ & $44.8(6.4)$ & $42.8(6.2)$ & 0.015 \\
\hline Weight, kg & $124(18)$ & $121(18)$ & $<0.001$ & $126(20)$ & $119(19)$ & 0.016 \\
\hline Waist circumference, $\mathrm{cm}$ & $127(13)$ & $123(13)$ & $<0.001$ & $127(14)$ & $123(13)$ & 0.025 \\
\hline WHR & $0.94(0.09)$ & $0.92(0.08)$ & $<0.001$ & $0.94(0.08)$ & $0.93(0.10)$ & 0.384 \\
\hline Metabolic syndrome & $370(60 \%)$ & 517 (53\%) & 0.008 & $157(68 \%)$ & $45(55 \%)$ & 0.032 \\
\hline -waist $\geq 80 \mathrm{~cm}$ & $611(100 \%)$ & $963(100 \%)$ & 1.000 & $228(100 \%)$ & $82(100 \%)$ & - \\
\hline -lipids & $457(74 \%)$ & $633(65 \%)$ & $<0.001$ & $186(81 \%)$ & $51(62 \%)$ & 0.001 \\
\hline triglycerides $\geq 1.7 \mathrm{mmol} / \mathrm{L}$ & $285(46 \%)$ & 398 (41 \%) & 0.037 & $116(50 \%)$ & $32(39 \%)$ & 0.094 \\
\hline $\mathrm{HDL}$-cholesterol $<1.30 \mathrm{mmol} / \mathrm{L}$ & $391(64 \%)$ & $469(49 \%)$ & $<0.001$ & $160(70 \%)$ & $41(50 \%)$ & 0.002 \\
\hline lipid lowering medication & $37(6 \%)$ & $66(7 \%)$ & 0.601 & $18(8 \%)$ & $8(10 \%)$ & 0.642 \\
\hline -raised blood pressure & $320(52 \%)$ & $570(59 \%)$ & 0.007 & $131(57 \%)$ & $50(61 \%)$ & 0.603 \\
\hline Systolic blood pressure $\geq 130, \mathrm{mmHg}$ & $233(38 \%)$ & $386(40 \%)$ & 0.428 & $106(47 \%)$ & $36(44 \%)$ & 0.700 \\
\hline Diastolic blood pressure $\geq 85, \mathrm{mmHg}$ & $195(32 \%)$ & $311(32 \%)$ & 0.868 & $74(33 \%)$ & $28(34 \%)$ & 0.785 \\
\hline Blood pressure lowering medication & $110(18 \%)$ & $246(25 \%)$ & 0.001 & $44(19 \%)$ & $17(21 \%)$ & 0.748 \\
\hline -fasting plasma glucose $\geq 5.6 \mathrm{mmol} / \mathrm{L}$ OR diabetes mellitus & $192(31 \%)$ & $270(28 \%)$ & 0.157 & $77(34 \%)$ & $23(28 \%)$ & 0.410 \\
\hline Type 2 diabetes & $112(18 \%)$ & $143(15 \%)$ & 0.069 & $42(18 \%)$ & $12(15 \%)$ & 0.501 \\
\hline Cholelithiasis & $59(10 \%)$ & $114(12 \%)$ & 0.187 & $18(8 \%)$ & $7(9 \%)$ & 0.816 \\
\hline Family history diabetes & $195(32 \%)$ & $300(31 \%)$ & 0.781 & $87(38 \%)$ & $30(37 \%)$ & 0.895 \\
\hline Systolic blood pressure, $\mathrm{mmHg}$ & $125(15)$ & $126(14)$ & 0.290 & $127(14)$ & $128(16)$ & 0.806 \\
\hline Diastolic blood pressure, $\mathrm{mmHg}$ & $80(10)$ & $80(10)$ & 0.394 & $80(10)$ & $80(10)$ & 0.516 \\
\hline Uric acid, $\mu \mathrm{mol} / \mathrm{L}$ & $345(69)$ & $325(69)$ & $<0.001$ & $358(69)$ & $325(72)$ & $<0.001$ \\
\hline $\mathrm{PTH}, \mathrm{pmol} / \mathrm{L}$ & $6.9(2.9)$ & $7.2(3.3)$ & 0.045 & $6.7(3.0)$ & $7.3(2.9)$ & 0.147 \\
\hline Vitamin D $25-\mathrm{OH}, \mathrm{nmol} / \mathrm{L}$ & $54(21)$ & $57(21)$ & 0.006 & $49(19)$ & $50(18)$ & 0.840 \\
\hline Magnesium & $0.84(0.07)$ & $0.85(0.07)$ & 0.178 & $0.83(0.07)$ & $0.83(0.07)$ & 0.978 \\
\hline Anxiety or depression & $290(47 \%)$ & $446(46 \%)$ & 0.643 & $122(53 \%)$ & $38(46 \%)$ & 0.306 \\
\hline Insulin, pmol/L & $129(95)$ & $107(83)$ & $<0.001$ & $142(75)$ & $118(70)$ & 0.012 \\
\hline HOMA IR, (mmol// * pmol/L / 135) & $5.7(6.0)$ & $4.5(4.4)$ & $<0.001$ & $6.0(3.9)$ & $4.8(3.4)$ & 0.017 \\
\hline$\geq 1$ hour physical activity/week & $329(64 \%)$ & 549 (67 \%) & 0.238 & $126(68 \%)$ & $44(60 \%)$ & 0.246 \\
\hline$\geq 1$ hour vigorous physical activity/week & $192(37 \%)$ & $356(43 \%)$ & 0.026 & $73(40 \%)$ & $32(44 \%)$ & 0.574 \\
\hline Use of estrogens or gestagen medication & $62(10 \%)$ & $112(12 \%)$ & 0.410 & $15(7 \%)$ & $20(24 \%)$ & $<0.001$ \\
\hline Smoke (current or former/never) & $368(60 \%)$ & $561(58 \%)$ & 0.432 & $147(64 \%)$ & $38(46 \%)$ & 0.006 \\
\hline $\mathrm{SHBG}, \mathrm{nmol} / \mathrm{L}$ & $22.3(9.2)$ & $44.3(29.2)$ & $<0.001$ & $20.8(8.1)$ & $46.6(30.3)$ & $<0.001$ \\
\hline Total testosterone, $\mathrm{nmol} / \mathrm{L}$ & $1.90(0.71)$ & $1.16(0.55)$ & $<0.001$ & $2.3(1.2)$ & $1.3(0.5)$ & $<0.001$ \\
\hline Estimated free testosterone, $\mathrm{nmol} / \mathrm{L}$ & $0.039(0.085)$ & $0.003(0.005)$ & $<0.001$ & $0.056(0.140)$ & $0.003(0.005)$ & 0.001 \\
\hline FTI & $1.3(3.1)$ & $0.3(0.1)$ & $<0.001$ & $1.7(5.2)$ & $0.3(0.1)$ & 0.017 \\
\hline
\end{tabular}

BMI; body mass index. WHR; waist hip ratio. PTH; parathyroid hormone. PCOS; polycystic ovarian syndrome. HOMA IR; Homeostasis Model Assessment - Insulin Resistance. FTI; free testosterone index, FTI > 0.6; Hyperandrogenemia (HA). SHBG; sex hormone binding globulin

HA was not associated with raised blood pressure $(1.06$ $[0.85,1.34])$. A sub-analysis of 1588 women without PCOS showed that HA remained significantly associated with MetS (OR 1.52 [95 \% CI 1.18, 1.96), dysglycemia (1.71
[1.30, 2.25]), low HDL-cholesterol (1.55 [1.22, 1.98]) and hypertriglyceridemia (1.36 [1.06, 1.74]) (Fig. 3B). In contrast, HA was neither significantly associated with MetS, nor its components in women with PCOS (Fig. 3C). 


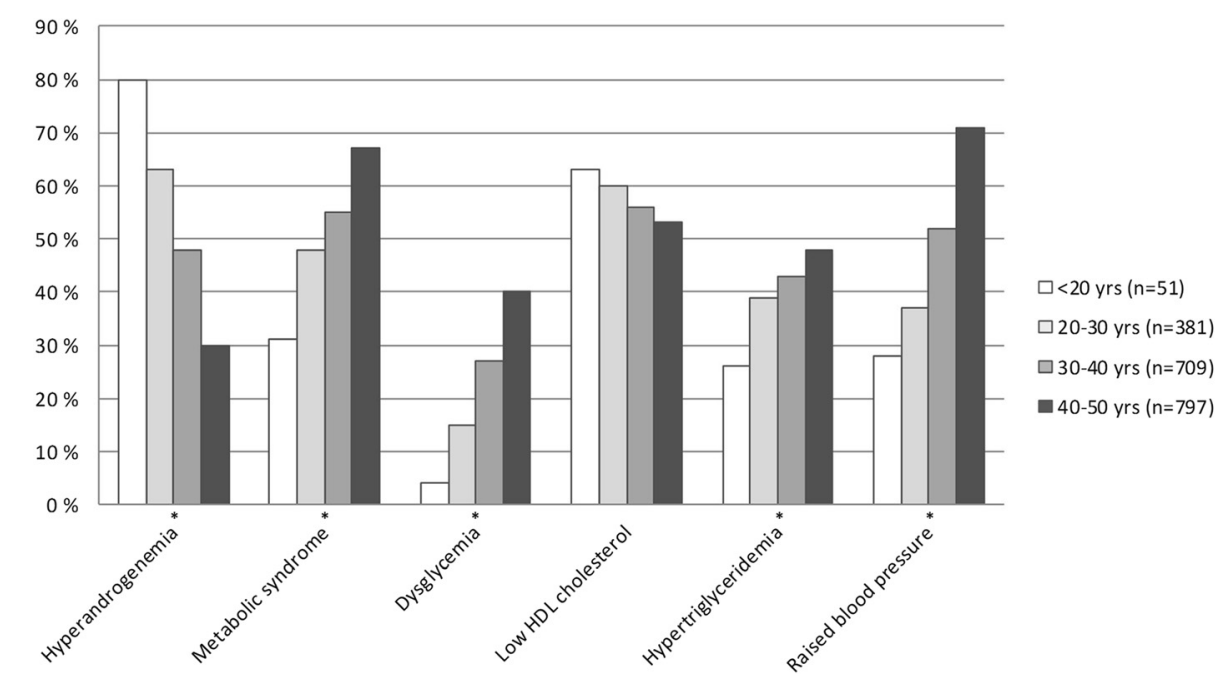

Fig. 2 The figure shows the proportion of morbidly obese women with hyperandrogenemia (HA), the metabolic syndrome (MetS) and its components according to various age categories. The prevalence of HA decreased, while the proportions of patients with MetS, dysglycemia, hypertriglyceridemia and raised blood pressure increased significantly with increasing age ( $\left.{ }^{*} p<0.001\right)$. The proportion of women with low HDL-cholesterol did, not change significantly with age $(p=0.145)$

\section{Discussion}

The main and novel finding of this study of premenopausal white morbidly obese women, was that HA was associated with $61 \%$ increased adjusted odds of MetS, and that this association was mainly driven by increased odds of dysglycemia and dyslipidemia. Although the prevalence of HA decreased, while the prevalence of MetS increased with increasing age, HA remained an independent predictor of MetS and its components, dysglycemia and dyslipidemia.

\section{Hyperandrogenemia and the metabolic syndrome}

The results of the present study cohere with another cross-sectional study of 2543 multiethnic overweight pre- and perimenopausal women who were on average age ten years older than the participants in the present study (46 vs. 37 years, respectively) [11]. In the previous study, women with HA, regardless of menstrual cycles, had a 1.5 fold increased risk of MetS compared to those without HA [11]. Women with both oligomenorrhea and HA had a 2 fold increased odds of MetS [11]. In our sub-analysis of 1588 severely obese women without PCOS, HA remained a significant predictor of MetS, dysglycemia, low HDL-cholesterol, and hypertriglyceridemia. In contrast, no association between androgen excess, menstrual irregularity and incident MetS or self-reported cardiovascular disease

Table 2 The odds of the metabolic syndrome (MetS) and its components adjusted for possible confounders.

\begin{tabular}{|c|c|c|c|c|c|c|c|c|c|c|}
\hline & \multicolumn{2}{|c|}{ Metabolic syndrome } & \multicolumn{2}{|c|}{ Dysglycemia } & \multicolumn{2}{|c|}{ Low HDL-cholesterol } & \multicolumn{2}{|c|}{ Hypertriglyceridemia } & \multicolumn{2}{|c|}{ Raised blood pressure } \\
\hline & OR & $95 \% \mathrm{Cl}$ & OR & $95 \% \mathrm{Cl}$ & OR & $95 \% \mathrm{Cl}$ & OR & $95 \% \mathrm{Cl}$ & OR & $95 \% \mathrm{Cl}$ \\
\hline Age, yrs & 1.06 & $1.04,1.07$ & 1.09 & $1.07,1.11$ & 1.00 & $0.98,1.01$ & 1.03 & $1.02,1.05$ & 1.08 & $1.07,1.10$ \\
\hline Family history diabetes & 1.41 & $1.12,1.77$ & 1.51 & $1.19,1.92$ & 1.24 & $0.99,1.54$ & 1.20 & $0.96,1.49$ & 1.36 & $1.09,1.71$ \\
\hline Uric acid, $\mu \mathrm{mol} / \mathrm{L}$ & 1.01 & $1.00,1.01$ & 1.00 & $1.00,1.00$ & 1.00 & $1.00,1.01$ & 1.01 & $1.00,1.01$ & 1.00 & $1.00,1.00$ \\
\hline Cholelithiasis & 1.52 & $1.05,2.21$ & 1.25 & $0.87,1.80$ & 1.16 & $0.82,1.64$ & 1.27 & $0.90,1.79$ & 1.24 & $0.87,1.78$ \\
\hline$\geq 1$ hour vigorous physical activity/week & 0.91 & $0.73,1.13$ & 0.95 & $0.75,1.20$ & 1.01 & $0.82,1.25$ & 0.81 & $0.66,1.00$ & 0.99 & $0.80,1.23$ \\
\hline Vitamin D-25-OH, nmol/L & 0.99 & $0.98,0.99$ & 0.99 & $0.98,0.99$ & 0.99 & $0.98,0.99$ & 1.00 & $0.99,1.00$ & 0.99 & $0.99,1.00$ \\
\hline $\mathrm{PTH}, \mathrm{pmol} / \mathrm{L}$ & 0.98 & $0.96,1.01$ & 0.98 & $0.96,1.01$ & 0.96 & $0.94,0.99$ & 0.97 & $0.95,1.00$ & 1.01 & $0.98,1.04$ \\
\hline Anxiety or depression & 1.33 & $1.08,1.65$ & 1.43 & $1.13,1.80$ & 1.22 & $0.99,1.50$ & 1.53 & $1.24,1.89$ & 0.91 & $0.73,1.12$ \\
\hline Smoke (current or former/never) & 1.45 & $1.17,1.79$ & 1.17 & $0.92,1.48$ & 1.38 & $1.12,1.70$ & 1.40 & $1.13,1.73$ & 0.97 & $0.78,1.20$ \\
\hline HA, FTI >0.6 (yes/no) & 1.61 & $1.27,2.02$ & 1.65 & $1.28,2.11$ & 1.58 & $1.27,1.97$ & 1.43 & $1.15,1.79$ & 1.06 & $0.85,1.34$ \\
\hline
\end{tabular}

Dependent variables; metabolic syndrome and its components. Hypertriglyceridemia; triglycerides; $\geq 1.7 \mathrm{mmol} / \mathrm{L}$, low HDL-cholesterol; HDL-cholesterol , $<1.3$ $\mathrm{mmol} / \mathrm{L}$, dysglycemia; fasting glucose $\geq 5.6 \mathrm{mmol} / \mathrm{L}$ or established diabetes mellitus, raised blood pressure; systolic blood pressure $\geq 130 \mathrm{mmHg}$ or diastolic blood pressure $\geq 85 \mathrm{mmHg}$ or use of blood pressure lowering medication. PTH; Parathyroid hormone. FTl; free testosterone index. HA; hyperandrogenemia. FTl; free testosterone index 



Fig. 3 (See legend on next page.) 
(See figure on previous page.)

Fig. 3 The figure shows the multivariable odds ratios with $95 \%$ confidence intervals (OR [95 \% Cl]) for metabolic syndrome (MetS) and its components in morbidly obese women with hyperandrogenemia compared to women without hyperandrogenemia (HA) (reference). Panel A comprises all the women included in the study $(n=1900)$, whereas Panel B and C comprises women included in the sub-analyses; PCOS absent $(n=1588)$ and PCOS present $(n=312)$

was revealed in a recent study of premenopausal women [28].

\section{Possible mechanisms linking hyperandrogenemia to insulin resistance and visceral obesity}

Animal (rodent) studies indicate that androgens may produce insulin resistance by direct effects on skeletal muscle and adipose tissue, mediated by alterations in the insulin receptor -glycogen synthesis, by altering adipokine secretion, and by increasing visceral adiposity [9]. Moreover, a small study of 13 obese and 30 non-obese women showed that anti-androgen treatment partly reversed the peripheral insulin resistance in non-obese women only, whereas central obesity may have a direct role in androgen hypersecretion [29,30]. Also, a recent study of young, overweight women suggested that the association between body fat and HA was predominantly mediated by insulin resistance [24]. The interrelationships between body fat, insulin resistance and HA contribute to the complex pattern making it a difficult task to specify the role of each component. Although the findings by Tosi et al. may not be directly comparable with ours, we also report a weak, but significant correlation between insulin resistance and HOMA IR and FTI. Acordingly, although HA was independently associated with MetS, insulin resistance might partly have mediated this effect.

\section{Association between hyperandrogenemia and dyslipidemia}

Our study demonstrated a significant inverse relationship between HA and HDL-cholesterol levels which is in accordance with previous studies of women with PCOS [31, 32]. Reduced HDL-cholesterol levels have also been reported in peri- and postmenopausal women receiving androgen formulations added to hormone replacement therapy and after transdermal testosterone treatment $[33,34]$. Furthermore, low levels of SHBG in women with PCOS have been associated with low levels of HDL-cholesterol, independent of insulin resistance and obesity [35]. Conversely, a recent study of young overweight women showed no differences in dyslipidemia in women with HA compared to women without HA [24]. Thus, the impact of HA on dyslipidemia remains unclear and further research is warranted.

\section{Hyperandrogenemia and dysglycemia}

The proportion of women with HA decreased, whereas the proportion of women with dysglycemia increased with age (Fig. 2). Nevertheless, we found a significant association between HA and dysglycemia independent of age.

Studies have shown a high conversion rate from impaired glucose tolerance to diabetes mellitus in women with PCOS [36, 37]. Our findings demonstrated that women with and without PCOS had a comparable prevalence of dysglycemia. However, patients with HA had approximately $65 \%$ increased odds of dysglycemia compared to those without $\mathrm{HA}$, and the association was strengthened after the exclusion of women with PCOS. These findings support the hypothesis that HA may be involved in the pathophysiology of dysglycemia independent of PCOS status.

\section{Hyperandrogenemia and blood pressure}

We did not find any significant association between HA and raised blood pressure. This is in contrast with the results from a study of young normal to overweight Asian women with PCOS which demonstrated that high bioavailable testosterone levels were associated with elevated blood pressure [38]. In another study of middle aged normal- to overweight women, facial hirsutism was associated with higher systolic blood pressure, whereas limb-hirsutism was associated with lower diastolic blood pressure [39]. In the present study, premenopausal morbidly obese women with HA were actually less likely to use blood pressure lowering medication compared to the women without HA (18 \% vs. $25 \%$ ). Interestingly, a recently published cross-sectional study of normal- to overweight Swedish men and women showed a strong inverse association between blood pressure and SHBG while free testosterone concentration was not associated with hypertension [40]. The authors speculated that SHBG might have a direct effect on the endothelial cells through the receptor for SHBG, but this association was significant only in postmenopausal women $\geq 50$ years of age [40]. In contrast, our study did not demonstrate any association between SHBG and raised blood pressure in premenopausal women (data not shown).

\section{Strengths and limitations}

The major strength of the present study is the large cohort of consecutively included treatment seeking morbidly obese women. The study participants were, however, referred to a 
tertiary care center for evaluation and treatment with bariatric surgery, medical therapy or long term lifestyle rehabilitation for morbid obesity. Accordingly, the results cannot be generalized to the general obese population (i.e. non-treatment seeking subjects).

We used an immunoassay to measure testosterone. Although conventional immunoassays measure reliable testosterone at high levels, the immunoassays have been reported to be less reliable at low concentrations [41]. However, the separation procedure of diethyl extraction was performed manually and should thus have reduced the number of falsely elevated results.

Furthermore, although we defined FTI $>0.6$ as HA in the present study, other adrenocortical precursor steroids including pregnenolone, 17-hydroxypregnenolone, dehydroepiandrosterone (DHEA), androstenedione, 11-deoxycortisol and cortisol may have contributed to clinical manifestations of HA. Nevertheless, free testosterone levels remain the predominant laboratory finding in women with oligomenorrhea and the ovaries are the main source of androgen excess in women with and without PCOS $[6,12]$.

Unfortunately, we did not have precise menopause data. There is some evidence that a higher BMI might cause a later menopause. One study reported that obese women (BMI $\geq 30 \mathrm{~kg} / \mathrm{m}^{2}$ ) had a median age at menopause of 53 years [17]. Consequently, by excluding women over 50 years of age, some morbidly obese premenopausal women might have been left out of our analysis.

Finally, the majority (97\%) of the morbidly obese women were Caucasian, and as such the results of might not be applicable to women of other ethnicities.

\section{Conclusion}

In this study, the prevalences of MetS, PCOS and HA were high among morbidly obese women $<50$ years of age. Compared to women without HA, those with HA had significantly higher odds of having the MetS, which was mainly explained by the associations between HA and the lipid- and glucose components of the MetS. Our results, if confirmed, suggest that an FTI-blood test might add value to the cardiovascular risk assessment of premenopausal women with morbid obesity. Randomized controlled clinical trials, including therapeutic strategies to lower free testosterone levels, are however necessary to explore any cause-and-effect relationship.

\footnotetext{
Abbreviations

BMI: Body mass index; WHR: Waist hip ratio; MetS: Metabolic syndrome; PCOS: Polycystic ovarian syndrome; HOMA IR: Homeostasis model assessment - insulin resistance; FTI: Free testosterone index; HA: Hyperandrogenemia; PTH: Parathyroid hormone.
}

\section{Competing interests}

The authors declare that they have no competing interests.

\section{Authors' contributions}

TGV and JH designed the study. JKH, JH, NN and DH helped collecting the data. TGV analyzed the data for the study. All authors contributed to the interpretation of the data. TGV drafted the manuscript. All authors reviewed and edited the manuscript. All authors gave their final approval of the final version to be published.

\section{Authors'information}

TGV is a clinical consultant and specialist in internal medicine at the Department of Endocrinology, Akershus University Hospital, Section for obesity and associated researcher at the Morbid Obesity Centre, Vestfold Hospital Trust, Tønsberg, Norway. JKH is a senior researcher and responsible for the obesity registry at Morbid Obesity Centre, Vestfold Hospital Trust, Tønsberg, Norway. NN is a clinical consultant and researcher at the Morbid Obesity Centre, Vestfold Hospital Trust, Tønsberg, Norway, specialized in cardiovascular disease and blood pressure in morbid obesity. POD is a senior researcher and Head of Department for Gynecology and Obstetrics at Vestfold Hospital Trust, Tønsberg, Norway, with experience from the field of $\mathrm{HA}$ and PCOS. DH is a consultant and clinical senior researcher at Morbid Obesity Centre, Vestfold Hospital Trust, Tønsberg, Norway. JH is a specialist in internal medicine and renal diseases. He heads both the multidisciplinary outpatient clinic and research group at the Morbid Obesity Centre, Vestfold Hospital Trust, Norway. He is also Professor at the Department of Endocrinology, Morbid Obesity and Preventive Medicine, the Medical Clinic, Institute of Clinical Medicine, University of Oslo, Norway.

\section{Acknowledgements}

We acknowledge the contribution of the staff at Morbid Obesity Centre, Vestfold Hospital Trust, for their persistent data collection efforts. Thanks are also due to Milada Småstuen for statistical advice and to Matthew McGee for proofreading the manuscript. All of the authors contributed according to the International Committee of Medical Journal Editors. No conflicts of interest were declared for any of the authors in the study.

\section{Author details}

${ }^{1}$ Morbid Obesity Centre, Vestfold Hospital Trust, Tønsberg, Norway. ${ }^{2}$ Division of Medicine, Department of Endocrinology, Akershus University Hospital HF, Sykehusveien 25, 1478 Nordbyhagen, Norway. ${ }^{3}$ Division of Medicine and Laboratory Sciences, Institute of Clinical Medicine, University of Oslo, Oslo, Norway. ${ }^{4}$ Department of Surgery, Vestfold Hospital Trust, Tønsberg, Norway. ${ }^{5}$ Department of Endocrinology, Morbid Obesity and Preventive Medicine, Institute of Clinical Medicine, University of Oslo, Oslo, Norway.

Received: 18 February 2015 Accepted: 5 May 2015

Published online: 21 May 2015

\section{References}

1. March WA, Moore VM, Willson KJ, Phillips DI, Norman RJ, Davies MJ. The prevalence of polycystic ovary syndrome in a community sample assessed under contrasting diagnostic criteria. Hum Reprod. 2010;25:544-51.

2. Yildiz BO, Knochenhauer ES, Azziz R. Impact of obesity on the risk for polycystic ovary syndrome. J Clin Endocrinol Metab. 2008;93:162-8.

3. Diamanti-Kandarakis E, Dunaif A. Insulin resistance and the polycystic ovary syndrome revisited: an update on mechanisms and implications. Endocr Rev. 2012;33:981-1030.

4. Alvarez-Blasco F, Botella-Carretero Jl, San Millan JL, Escobar-Morreale HF. Prevalence and characteristics of the polycystic ovary syndrome in overweight and obese women. Arch Intern Med. 2006;166:2081-6.

5. Teede H, Deeks A, Moran L. Polycystic ovary syndrome: a complex condition with psychological, reproductive and metabolic manifestations that impacts on health across the lifespan. BMC Med. 2010;8:41.

6. Azziz R, Carmina E, Dewailly D, Diamanti-Kandarakis E, Escobar-Morreale HF, Futterweit W, et al. The Androgen Excess and PCOS Society criteria for the polycystic ovary syndrome: the complete task force report. Fertil Steril. 2009:91:456-88.

7. Lim SS, Norman RJ, Davies MJ, Moran LJ. The effect of obesity on polycystic ovary syndrome: a systematic review and meta-analysis. Obes Rev. 2013;14:95-109.

8. Nestler JE, Powers LP, Matt DW, Steingold KA, Plymate SR, Rittmaster RS, et al. A direct effect of hyperinsulinemia on serum sex hormone-binding 
globulin levels in obese women with the polycystic ovary syndrome. J Clin Endocrinol Metab. 1991;72:83-9.

9. Holmang A, Larsson BM, Brzezinska Z, Bjorntorp P. Effects of short-term testosterone exposure on insulin sensitivity of muscles in female rats. Am J Physiol. 1992;262:E851-5

10. Nohara K, Laque A, Allard C, Munzberg H, Mauvais-Jarvis F. Central mechanisms of adiposity in adult female mice with androgen excess. Obesity (Silver Spring). 2014;22:1477-84

11. Polotsky AJ, Allshouse A, Crawford SL, Harlow SD, Khalii N, Santoro N, et al. Relative contributions of oligomenorrhea and hyperandrogenemia to the risk of metabolic syndrome in midlife women. J Clin Endocrinol Metab. 2012;97:E868-77.

12. Ehrmann DA. Polycystic ovary syndrome. N Engl J Med. 2005;352:1223-36.

13. Lachelin GC, Barnett M, Hopper BR, Brink G, Yen SS. Adrenal function in normal women and women with the polycystic ovary syndrome. J Clin Endocrinol Metab. 1979;49:892-8.

14. Wild RA, Carmina E, Diamanti-Kandarakis E, Dokras A, Escobar-Morreale HF, Futterweit W, et al. Assessment of cardiovascular risk and prevention of cardiovascular disease in women with the polycystic ovary syndrome: a consensus statement by the Androgen Excess and Polycystic Ovary Syndrome (AE-PCOS) Society. J Clin Endocrinol Metab. 2010;95:2038-49.

15. Chang AY, Ayers C, Minhajuddin A, Jain T, Nurenberg P, de Lemos JA, et al. Polycystic ovarian syndrome and subclinical atherosclerosis among women of reproductive age in the Dallas heart study. Clin Endocrinol (Oxf). 2011;74:89-96.

16. Moran $L$, Teede H. Metabolic features of the reproductive phenotypes of polycystic ovary syndrome. Hum Reprod Update. 2009;15:477-88.

17. Morris DH, Jones ME, Schoemaker MJ, McFadden E, Ashworth A, Swerdlow AJ. Body mass index, exercise, and other lifestyle factors in relation to age at natural menopause: analyses from the breakthrough generations study. Am J Epidemiol. 2012;175:998-1005.

18. Overlie I, Moen MH, Morkrid L, Skjaeraasen JS, Holte A. The endocrine transition around menopause-a five years prospective study with profiles of gonadotropines, estrogens, androgens and SHBG among healthy women. Acta Obstet Gynecol Scand. 1999;78:642-7.

19. Wallace WH, Kelsey TW. Human ovarian reserve from conception to the menopause. PLoS One. 2010;5, e8772.

20. World Medical Association Declaration of Helsinki. ethical principles for medical research involving human subjects. JAMA. 2000;284:3043-5.

21. Alberti KG, Eckel RH, Grundy SM, Zimmet PZ, Cleeman Jl, Donato KA, et al. Harmonizing the metabolic syndrome: a joint interim statement of the International Diabetes Federation Task Force on Epidemiology and Prevention; National Heart, Lung, and Blood Institute; American Heart Association; World Heart Federation; International Atherosclerosis Society; and International Association for the Study of Obesity. Circulation. 2009;120:1640-5

22. Matthews DR, Hosker JP, Rudenski AS, Naylor BA, Treacher DF, Turner RC Homeostasis model assessment: insulin resistance and beta-cell function from fasting plasma glucose and insulin concentrations in man. Diabetologia. 1985;28:412-9

23. Vermeulen A, Verdonck L, Kaufman JM. A critical evaluation of simple methods for the estimation of free testosterone in serum. J Clin Endocrinol Metab. 1999:84:3666-72.

24. Tosi F, Di SD, Kaufman JM, Bonin C, Moretta R, Bonora E, et al. Total body fat and central fat mass independently predict insulin resistance but not hyperandrogenemia in women with polycystic ovary syndrome. J Clin Endocrinol Metab. 2014;2:661-9.

25. Free testosterone indeks (FTI) in serum. Hormone Laboratory Oslo University Hospital Aker. http://www.oslouniversitetssykehus.no/omoss_/avdelinger_/ hormonlaboratoriet_/analyser_/Sider/fri-testosteron-indeks-i-serumfti.aspx. Accessed 15-12-2014.

26. Rotterdam ESHRE/ASRM-Sponsored PCOS consensus workshop group. Revised 2003 consensus on diagnostic criteria and long-term health risks related to polycystic ovary syndrome (PCOS). Hum Reprod. 2004;19:41-7.

27. Monge M, Jean G, Bacri JL, Lemaitre V, Masy E, Joly D, et al. Higher parathyroid hormone (PTH) concentrations with the Architect PTH assay than with the Elecsys assay in hemodialysis patients, and a simple way to standardize these two methods. Clin Chem Lab Med. 2009:47:362-6.

28. Polotsky AJ, Allshouse AA, Crawford SL, Harlow SD, Khalil N, Kazlauskaite R, et al. Hyperandrogenic oligomenorrhea and metabolic risks across menopausal transition. J Clin Endocrinol Metab. 2014;99:2120-7.
29. Moghetti P, Tosi F, Castello R, Magnani CM, Negri C, Brun E, et al. The insulin resistance in women with hyperandrogenism is partially reversed by antiandrogen treatment: evidence that androgens impair insulin action in women. J Clin Endocrinol Metab. 1996:81:952-60.

30. Diamanti-Kandarakis E. Role of obesity and adiposity in polycystic ovary syndrome. Int J Obes (Lond). 2007;31 Suppl 2:S8-13.

31. Apridonidze T, Essah PA, luorno MJ, Nestler JE. Prevalence and characteristics of the metabolic syndrome in women with polycystic ovary syndrome. J Clin Endocrinol Metab. 2005;90:1929-35.

32. Legro RS, Urbanek M, Kunselman AR, Leiby BE, Dunaif A. Self-selected women with polycystic ovary syndrome are reproductively and metabolically abnormal and undertreated. Fertil Steril. 2002;78:51-7.

33. Somboonporn W, Davis S, Seif MW, and Bell, R. Testosterone for peri- and postmenopausal women. http://onlinelibrary.wiley.com/doi/10.1002/ 14651858.CD004509.pub2/pdf

34. Wang X, Smith Gl, Patterson BW, Reeds DN, Kampelman J, Magkos F, et al. Testosterone increases the muscle protein synthesis rate but does not affect very-low-density lipoprotein metabolism in obese premenopausal women. Am J Physiol Endocrinol Metab. 2012;302:E740-6.

35. Chen MU, Yang WS, Yang JH, Hsiao CK, Yang YS, Ho HN. Low sex hormone-binding globulin is associated with low high-density lipoprotein cholesterol and metabolic syndrome in women with PCOS. Hum Reprod. 2006;21:2266-71.

36. Norman RJ, Masters L, Milner CR, Wang JX, Davies MJ. Relative risk of conversion from normoglycaemia to impaired glucose tolerance or non-insulin dependent diabetes mellitus in polycystic ovarian syndrome. Hum Reprod. 2001;16:1995-8.

37. Vrbikova J, Dvorakova K, Grimmichova T, Hill M, Stanicka S, Cibula D, et al. Prevalence of insulin resistance and prediction of glucose intolerance and type 2 diabetes mellitus in women with polycystic ovary syndrome. Clin Chem Lab Med. 2007;45:639-44.

38. Chen MJ, Yang WS, Yang JH, Chen $\mathrm{CL}$, Ho HN, Yang YS. Relationship between androgen levels and blood pressure in young women with polycystic ovary syndrome. Hypertension. 2007;49:1442-7.

39. Muti P, Trevisan M, Panico S, Micheli A, Celentano E, Freudenheim JL, et al. Body fat distribution, peripheral indicators of androgenic activity, and blood pressure in women. Ann Epidemiol. 1996;6:181-7.

40. Daka B, Rosen T, Jansson PA, Larsson CA, Rastam L, Lindblad U. Low sex hormone-binding globulin is associated with hypertension: a cross-sectional study in a Swedish population. BMC Cardiovasc Disord. 2013;13:30.

41. Roy P, Alevizaki M, Huhtaniemi I. In vitro bioassays for androgens and their diagnostic applications. Hum Reprod Update. 2008;14:73-82.

\section{Submit your next manuscript to BioMed Central and take full advantage of:}

- Convenient online submission

- Thorough peer review

- No space constraints or color figure charges

- Immediate publication on acceptance

- Inclusion in PubMed, CAS, Scopus and Google Scholar

- Research which is freely available for redistribution 